\title{
DOCENCIA Y ACCIÓN TUTORIAL EN LA EDUCACIÓN TECNOLÓGICA SUPERIOR: EXPERIENCIA Y REFLEXIONES.
}

Autor.

Rafael F. Bell

Profesor Titular del ITF

rafael.bell@formacion.edu.ec
Coautor.

Daniel A. Zaldívar

Profesor Titular del ITF

daniel.zaldivar@formacion.edu.ec
Coautor.

Alicia V. Alvarez

Profesora Titular del ITF

alicia.alvarez@formacion.edu.ec
País Origen: Ecuador

Palabras claves: Docencia, tutorias, acción tutorial, titulación
Recibido: 11 de Marzo del 2016

Aceptado: 15 de Abril del 2016

Keywords: Teaching, mentoring, certification

\section{SUMMARY}

$\mathrm{T}$

he present article deals with the challenges faced by professors of the higher education institutions nowadays, taking into consideration the demands caused by the constant development of the information and communication technologies, at the same time by the complexity of the different phenomena that are part of the daily life.

Having this as basic ground, the main guidelines in order to resize the functions of professors are established in the article , particularly those related to the teaching -learning process, and more specifically with the mentoring called to become a privileged space for interaction and growth of both , students and professors .

\section{RESUMEN}

E n este artículo se reflexiona en torno a los retos a los que se enfrenta la labor docente del profesorado de las Instituciones de la Educación Tecnológica Superior en la actualidad, a la luz de las exigencias que se derivan del incesante desarrollo de las tecnologías de la información y de las comunicaciones y de la creciente complejidad de los hechos y fenómenos que configuran el diario quehacer de la humanidad.

En este contexto se exponen las principales pautas para redimensionar las funciones del profesorado, en particular las relacionadas con la docencia y más concretamente con las tutorias, llamadas a convertirse en un espacio privilegiado para la interacción y el crecimiento conjunto de estudiantes y profesores.

la viabilidad en el ingreso y la promoción de la carrera 


\section{INTRODUCCIÓN}

a sociedad del conocimiento caracterizada, entre otros factores, por el incesante desarrollo de las Tecnologías de la Información y de las Comunicaciones (TIC) y por la alta producción del conocimiento unida a su pronta obsolescencia, implica crecientes desafíos para la formación de las nuevas generaciones y coloca las funciones del profesorado como una de las cuestiones que mayor atención reclama pues de ella depende, en gran medida, el necesario avance de la educación en el siglo XXI.

Este reto adquiere matices particulares en la educación tecnológica superior en la que se requiere conjugar la sólida formación y experiencia de los profesores en sus perfiles profesionales con una imprescindible preparación pedagógica y didáctica como fundamento para el mejor desempeño de sus funciones educativas en las condiciones actuales.

En consonancia con ello, el presente artículo pretende contribuir a promover el análisis y la reflexión en torno a las funciones del profesorado en la educación tecnológica superior, con énfasis en la función docente y en la comprensión del papel que juegan las tutorías como una indispensable herramienta para el logro de los fines y objetivos de este tipo de institución.

\section{DESARROLLO}

Las profundas transformaciones sociales que tienen lugar en el mundo actual relacionadas, entre otros factores, con la globalización, el indetenible desarrollo de las TIC, la creciente complejidad de los fenómenos y situaciones de la realidad, entre otras, repercuten en todos los ámbitos y esferas de la actividad humana y la educación no constituye una excepción.

Por el contrario, se podría afirmar que en el ámbito educativo las repercusiones de las referidas transformaciones adquieren una connotación particular, pues su impacto plantea la necesidad de revisar, actualizar y cambiar muchas de las ideas y propuestas educativas consideradas hasta no hace mucho tiempo como fundamentos de las prácticas establecidas, que con frecuencia se asumían con carácter prácticamente inalterable.

Desde esa perspectiva pueden ser valoradas las funciones del profesorado de la educación superior, que habitualmente se enmarcan en cuatro grupos fundamentales:

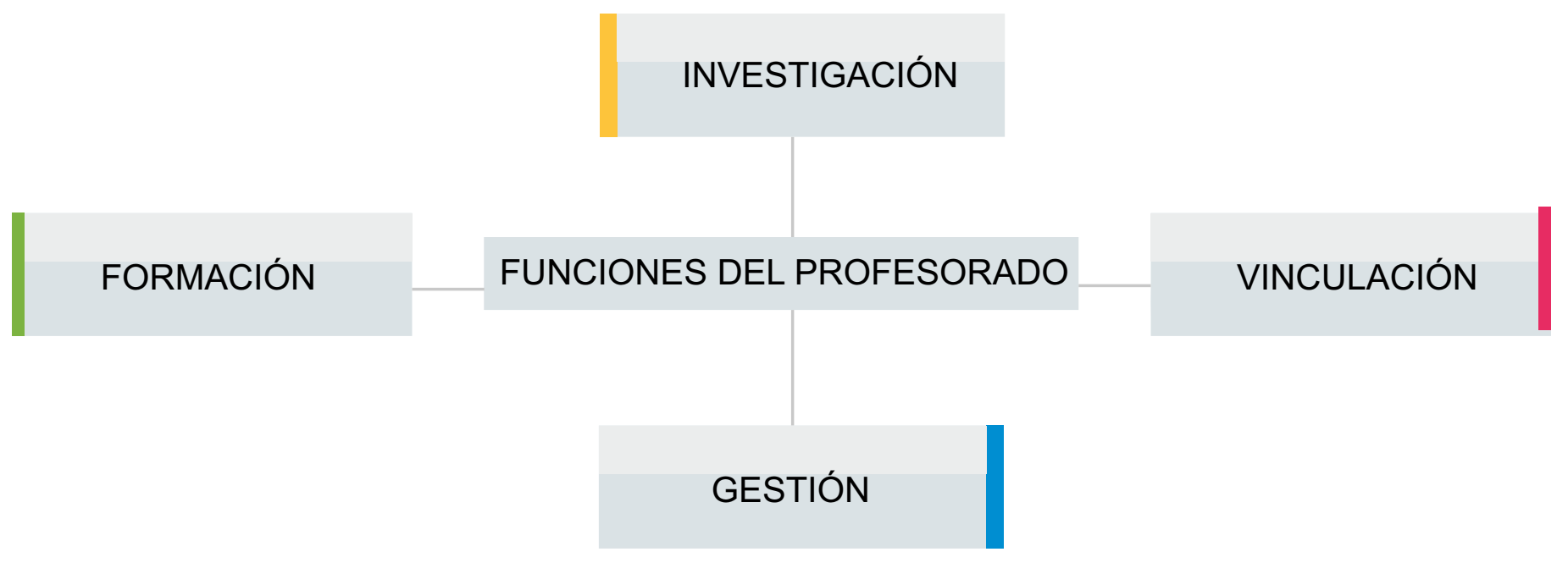

Docencia-Investigación-Vinculación-Gestión

Sin embargo, la necesaria articulación entre los referidos grupos de funciones del profesorado todavía se encuentra distante de mostrar la interrelación y armonía requeridas para un desempeño integralmente exitoso de los profesores, que al mismo tiempo se ha de convertir en un modo de actuación que sus estudiantes pueden asumir como modelo para el mejor cumplimiento de sus actividades durante su etapa de formación en la educación superior. 


\section{Docencia en las Instituciones de Educación Superior (IES)}

En el marco de este análisis no hay que obviar que habitualmente se suele colocar un mayor énfasis en la función del profesorado vinculada con la docencia, aunque ella, en muchos casos, se mantiene atrapada en los límites de la transmisión de conocimientos. Pero hoy se alcanza un consenso cada vez mayor acerca de la necesidad de rebasar esa postura, pues como precisan Rajadell y Medina (2009) "enseñar ya no equivale sólo a transmitir una materia" (p.93).

Por consiguiente se impone la necesidad de redimensionar la función docente del profesor de la Educación Superior y en ese empeño se pueden asumir como pautas para la acción encaminada a la renovación docente, las ideas que Zabalza (2009) resume de la siguiente manera:

- El reconocimiento de la importancia de la docencia como eje vertebrador de la formación y de la actividad en general de las IES.

- La comprensión de las características propias de la docencia pues siendo el vaso comunicante de la labor universitaria, mantiene sus rasgos propios, que la distinguen de la investigación, de la vinculación y de la gestión.

- La importancia del desarrollo de una labor docente acompañada de la reflexión y de la formación como vías para alcanzar una mejor respuesta a las exigencias, condiciones y propósitos de la formación.

- El establecimiento de la docencia como un espacio propio en el que se integran los conocimientos, las habilidades y las actitudes que le han de ser inherentes a todos los formadores.

Estas ideas, unidas al análisis de las mejores experiencias de la labor del profesorado de las IES en las condiciones actuales y a las exigencias derivadas de los cambios de enfoques en el contexto pedagógico, se convierten en el punto de partida para la promoción del redimensionamiento de la función docente de los profesores, llamados a convertirse más que en transmisores del conocimiento, en generadores de condiciones y en guías para nuevos aprendizajes.

De esta manera y de acuerdo con Cano (2009) ahora al profesorado le corresponde asumir que el estudiante debe convertirse en el centro del proceso de enseñanza-aprendizaje, en cuyo desarrollo ha de aprender haciendo y para ello el docente recurrirá a formas de enseñar que motiven y guíen el aprendizaje de todo el alumnado.

Consiguientemente se hace necesario alcanzar una mayor claridad en torno a las funciones que se integran para el adecuado desempeño docente del profesorado de las IES, incluidos los Institutos Tecnológicos Superiores. Así, entre las referidas funciones se pueden resaltar, a partir de lo señalado por Zabalza (2003), las siguientes:

- Adecuada planificación del proceso de enseñanza-aprendizaje.

- Cuidadosa selección y preparación de los contenidos de las diferentes materias.

- Diseño y aplicación de metodologías didácticas y organizativas que propicien el aprendizaje, la actividad conjunta y la comunicación.

- Dominio y aprovechamiento de las potencialidades de las TIC para la promoción de un aprendizaje activo y significativo.

- Aplicación de diversas formas y modalidades de evaluación

- Desarrollo de un enfoque reflexivo e investigativo en relación con la labor docente.

- Fomento de la capacidad para el trabajo en equipo.

- Seguimiento a los syllabus de las diferentes materias.

- Realización de las tutorías.

El exitoso cumplimiento de estas funciones ha de servir como soporte para potenciar la interdependencia entre la docencia y la acción tutorial, que constituyen la esencia de la formación como función del profesorado, lo que se refleja en el siguiente esquema: 


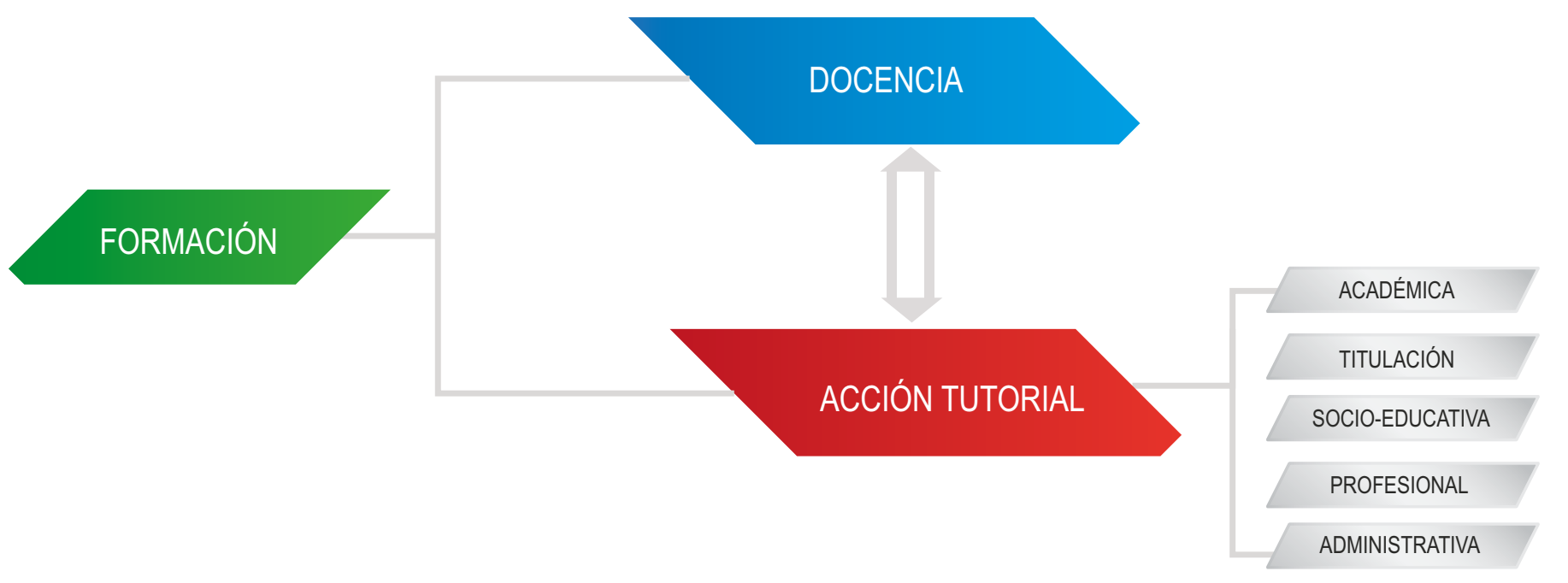

\section{Docencia-Investigación-Vinculación-Gestión}

La trascendencia e interrelación directa de la actividad docente con las tutorías justifican la necesidad de brindar a las mismas una atención particular, lo que a continuación será objeto de reflexión y análisis.

\section{Acción tutorial en las IES}

La presencia de una diversidad de población adulta en las IES constituye uno de los principales rasgos distintivos de la educación superior y plantea importantes desafíos para el desempeño de los docentes, en concreto, en relación con las tutorías, cuyo desarrollo se asocia por lo general con las etapas educativas previas y no siempre se valora de la misma manera la importancia de su realización en los niveles superiores de educación.

Al parecer existe cierta creencia acerca de que dada la condición de adultos de los estudiantes de las IES no se hace indispensable prestar a las tutorías una atención priorizada, aun cuando los estudiantes, al igual que toda la sociedad, se encuentran expuestos a crecientes riesgos y amenazas, derivados del agravamiento de las situaciones sociales y familiares, de la agudización de las manifestaciones de agresividad y violencia a nivel social y familiar, de los serios problemas para el empleo y de las enfermedades de transmisión sexual con sus severas consecuencias, entre otros.

Lógicamente la búsqueda de respuestas y soluciones a una problemática tan compleja no depende exclusivamente de las IES ni de la labor del profesorado, pero sin su contribución es difícil imaginar que se pueda avanzar al ritmo que la urgencia del tema exige. En ese empeño las tutorías en la educación superior están llamadas a realizar, como indican Nieto, Muñoz, Santaolalla, García y González (2007) una importante contribución, como "medio para estimular, guiar, apoyar y evaluar el aprendizaje y desarrollo integral del estudiante" (p. 17).

Sin embargo, habitualmente la referencia a la tutoría universitaria se asocia, en primera instancia, con la labor de asesoramiento, orientación y revisión de los trabajos de titulación de los estudiantes, que continúa siendo una modalidad fundamental de la acción tutorial, pero que no agota su riqueza y alcance.

Ante la complejidad de la situación esbozada, en la que también está presente la variedad de enfoques, definiciones y tipos de tutorías parece conveniente adoptar determinados referentes conceptuales como coordenadas preliminares que favorezcan una mejor aproximación al tema objeto de estudio.

En este sentido resulta de interés esbozar algunas de las definiciones y características más relevantes de las tutorías que aparecen en las fuentes bibliográficas consultadas. Así, por ejemplo, Gairín, Feixas, Guillamón, y Quinquer (2004) definen la acción tutorial como un "proceso orientador que desarrollan de manera conjunta profesor y estudiante en aspectos académicos, profesionales y personales con la finalidad de establecer un programa de trabajo que favorezca la confección y diseño de la trayectoria más adecuada a la carrera seleccionada"(p.67).

Ferrer (2003) destaca el carácter formativo de las tutorías y sus repercusiones en el desarrollo de diversas aristas del desarrollo integral de la personalidad de los estudiantes, entre las que se incluyen las esferas cognitiva, académica, profesional y del desarrollo a nivel personal.

A su vez, García Nieto, Asensio, Carballo, García García y Guardia (2005) insisten en las tutorías como uno de los ámbitos de responsabilidad del docente y destacan que en las mismas se establece una interacción más directa, 
de tipo personalizada, entre el docente y el estudiante con el propósito de ofrecer las orientaciones y pautas que favorezcan su mejor desempeño académico, profesional, administrativo, social y personal.

Al hilo de lo señalado no es difícil advertir la enorme connotación que las tutorías tienen para la labor de las IES, pues generan un privilegiado espacio de interacción y aprendizaje con amplias potencialidades para ejercer su influencia en diversas áreas del desarrollo de los estudiantes, en particular en la académica, la profesional y la personal.

Sin embargo, en la experiencia de las IES y en concreto, de los Institutos Tecnológicos Superiores, todavía se aprecian determinadas limitaciones y dificultades en la concepción y desarrollo de las tutorías, entre las cuales se pueden distinguir las siguientes:

- Marcada tendencia a la asociación de la tutoría, casi de manera exclusiva, al proceso de titulación de los estudiantes.

- Irregularidades en la planificación de los horarios para la realización de las tutorías.

- Falta de puntualidad e inasistencia de los estudiantes a sus sesiones de tutorías.

- Déficits en la preparación del profesorado que no siempre domina las herramientas metodológicas para brindar a cada estudiante, en las tutorías, la atención que requiere.

- Carencia de recursos metodológicos para una mejor preparación de los docentes para la realización de las tutorías.

- Escaso interés de las investigaciones por las tutorías, que todavía no constituyen un objeto priorizado de estudio.

- Dificultades en el uso intensivo de las TIC como una herramienta cuya aplicación efectiva ha de potenciar el desarrollo de la acción tutorial.

Ante esta realidad se hace imprescindible reforzar la labor de las IES en torno al reconocimiento del papel de las tutorías, comenzando por las de tipo académico y sobre todo las relacionadas con los proyectos de titulación para avanzar, al mismo tiempo, hacia el establecimiento y generalización de las tutorías, en todas sus dimensiones y modalidades, como un requerimiento y una actividad indispensable para alcanzar el cumplimiento de los objetivos previstos.

\section{Tutorías de trabajos de titulación: experiencias y reflexiones}

En la planificación y realización de las tutorías de los trabajos de titulación es necesario considerar diversos factores entre los cuales se encuentran la cantidad de alumnos que deben acceder a las tutorías, sus características individuales, la simultaneidad de esta actividad con otras inherentes a la actividad formativa, en particular la docencia y la investigación, la experiencia y características del tutor y los requerimientos propios de cada carrera y de cada tipo de trabajo de titulación.

En relación con este último aspecto cabe apuntar la diversidad de opciones de trabajos de titulación contempladas en el Reglamento de Régimen Académico, en el que, entre otras, se contemplan el examen de grado o de fin de carrera, los proyectos de investigación, los proyectos integradores, ensayos a artículos académicos, etnografías, análisis de casos, estudios comparados, modelos de negocios, emprendimientos, etc.

A la luz de los elementos señalados con anterioridad al tutor le corresponde planificar las tutorías, lo que, en la experiencia desarrollada, implicó, en primer lugar, el establecimiento de cronogramas de trabajo, en horarios accesibles para el estudiante, y para el tutor, teniendo en cuenta la variedad de horarios en los que el profesor está comprometido con la docencia. En consecuencia, los cronogramas fueron establecidos de forma grupal y de forma individual con la intención de satisfacer las exigencias y requerimientos expuestos.

De igual manera el tutor realizó un diagnóstico previo, alumno por alumno, del grado de conocimiento, capacidades y competencias para asumir el trabajo de titulación propuesto, con la finalidad de delimitar el área del conocimiento que el estudiante domina y cual no, lo que permite acometer acciones individualizadas para mejorar los aspectos en los que el estudiante presenta carencias en el dominio de la metodología de la investigación o en diferentes aspectos relacionados con el tema de su trabajo. 
En cuanto a los agrupamientos, en el inicio se realizaron tutorías grupales, considerando los estudiantes de la carrera de Tecnología Superior en Comercio Exterior que tenían temas comunes, como los 6 temas relacionados con las exportaciones, para facilitar la comunicación tutor- estudiante y estudiante- estudiante, creando de esta manera un circulo de estudio y cooperación, que facilitó mucho la labor del tutor, y el intercambio de conocimientos entre los estudiantes con temas de la misma área de estudio.

También se analizó el grado de motivación y compromiso de cada estudiante, su entorno socioeconómico y se detectaron las posibles causas que pudieran afectar el rendimiento y el compromiso con su investigación. Evidentemente el acercamiento a cada alumno permite conocer mejor su entorno y conocer sus particularidades.

Luego, a medida que se fue avanzando en temas comunes, se logró personalizar cada tutoría, en la que se revisaban los señalamientos anteriores y el grado de avance de cada estudiante y se dejaba constancia de los elementos que era necesario reconsiderar para la próxima revisión.

Los cronogramas de trabajo no fueron rígidos, fueron flexibles, teniendo en cuenta las dificultades que presentan los estudiantes: algunos viven en zonas lejanas, otros presentan dificultades económicas. Por ello se decidió aprovechar las bondades que ofrecen las TIC en la actualidad. Así, se pasó de un ambiente físico, de contacto personal en las primeras semanas a un ambiente virtual, cuestión que ayuda a ganar en tiempo y comunicación, por tanto, favorece el proceso de tutoría a partir del uso de las siguientes herramientas y aplicaciones:,

- Skype: para videoconferencia, ya fuera grupal o individual.

- Whatsap: se creó un grupo específico, para estudiantes en tutorías, muy útil por el carácter instantáneo de ese protocolo de mensajería y la inmediatez de la información.

- Google Drive: para compartir documentos, bibliografía, y las herramientas disponibles en Google.

- Correo electrónico: para la comunicación sistemática y de una manera más detallada con los estudiantes.

Con relación a la orientación metodológica se trabajó con los estudiantes en la comprensión y manejo de bases de datos internacionales como:

- Base mundial de datos sobre Marcas

- Consulta TARIC de la Unión Europea

- Einforma

- Euromonitor Internacional

- TradeMap

- Kompass

- Romarin

- Tariffdata

Se insistió en que el uso de las referidas bases de datos les permitiría la obtención de informaciones de referencia a nivel internacional, validada por organismos competentes, lo que brindaría un sólido sustento a sus trabajos.

Al unísono se comenzó a ampliar y a personalizar la bibliografía de consulta, utilizando los fundamentos legales comunes y precisando las fuentes específicas de mayor connotación para cada tema. Al respecto se trabajó en la comprensión por los estudiantes de la importancia de una bibliografía de calidad, actualidad y de elevado rigor científico, lo que se convierte en un respaldo del trabajo que realiza, le ayuda a superar el grado de duda y a confirmarle que está siendo objetivo con lo que se investiga.

Es este aspecto es importante lograr que los estudiantes identifiquen y reconozcan a los principales autores de referencia en relación con su tema, las obras publicadas, trabajos, artículos, etc. pues de ese modo estará en mejores condiciones para discernir sobre la gran cantidad de información que se encuentra disponible en Internet, discriminando aquella que pueda confundir o ser la base de una investigación sin fundamento. Pueden ser resumidas de la de manera siguiente: 
En la etapa final la acción tutorial se enfocó a la preparación de los estudiantes para la sustentación y defensa del trabajo de titulación. En esta fase se le prestó una particular atención al dominio del tema, al contenido de la información que se proyectaría como apoyo a la presentación, a las diferentes expresiones orales, corporales y en general al desarrollo de las competencia comunicativa tanto verbal como no verbal para un adecuado desempeño ante el tribunal.

Durante las distintas fases se mantuvo un seguimiento de las tutorías mediante la aplicación del módulo "docentes" del Sistema de Gestión Académica del Instituto, lo que favoreció el registro de los aspectos más relevantes del trabajo, incluida la aprobación o no por parte de los estudiantes de las tutorías recibidas.

El cumplimiento de las tutorías de los trabajos de titulación con la metodología presentada durante este período académico permitió identificar las principales dificultades y limitaciones de los estudiantes para asumir con mayor nivel de éxito la elaboración y presentación de sus trabajos, las que pueden ser resumidas de la de manera siguiente:

1. No se encuentran desarrolladas al nivel requerido las competencias de los estudiantes para el análisis y la comprensión de contenidos de diversas fuentes de información, situación que se agrava por sus limitaciones para distinguir entre una fuente de información correcta y una incorrecta o de poco valor.

2. El dominio de la metodología de la investigación es todavía insuficiente y en algunos casos se aprecia la tendencia a considerarla de manera superflua o a subestimarla.

3. La presentación del marco legal de los trabajos de titulación casi siempre se asume de manera mecánica y no se estructura en el orden jerárquico correcto. Por consiguiente se tiende a analizar solo la norma de inferior jerarquía, no la estructura legal que ampara el trabajo.

4. Aún no se aprovechan de manera óptima los recursos y herramientas tecnológicas en función de los trabajos de titulación.

5. Dificultades de redacción y ortografía, lo que compromete la calidad y valoración integral del trabajo.

Sin embargo, mediante la aplicación de la metodología prevista fue posible brindar la atención que cada una de las dificultades señaladas demandaba y ello hizo posible que todos los estudiantes lograran vencer las metas planteadas para cada etapa, lo que los condujo al cumplimiento exitoso del objetivo de la titulación.

\section{CONCLUSIONES}

Las actuales condiciones del desarrollo de la sociedad y los desafíos a los que en ese contexto se enfrentan las IES implican la necesidad del redimensionamiento de las funciones del profesorado en la Educación Superior y en ese sentido se hace imprescindible colocar un énfasis particular en el papel de las tutorías, cuya correcta comprensión y aplicación encierra grandes ventajas potenciales para repercutir en la vida académica, personal, social y profesional del alumnado.

Una de las vías a las que se recomienda recurrir está relacionada con las tutorías de los trabajos de titulación, de cuya realización se pueden extraer valiosas conclusiones para el mejor desempeño integral de los estudiantes y para la acción tutorial en general.

En este sentido resultará imprescindible redoblar los esfuerzos en la formación del profesorado para las tutorías, en la elaboración de materiales metodológicos para una mejor orientación de la actividad de los tutores en diferentes ámbitos, tomando como punto de partida el relacionado con los trabajos de titulación y el reconocimiento y la difusión de las mejores experiencias de los tutores. 


\section{BIBLIOGRAFÍA}

Cano, R. (2009). Tutoría universitaria y aprendizaje por competencias ¿Cómo lograrlo? Revista Electrónica Interuniversitaria de Formación del Profesorado, 12(1), 181-204.

Ferrer, V. (2003). La acción tutorial en la Universidad. En F. Michavila \& J. García (coords.), La Tutoría y los nuevos modelos de aprendizaje en la Universidad (pp. 67-84). Madrid: Consejería de Educación de la Comunidad Autónoma de Madrid/Cátedra UNESCO de Gestión Política Universitaria de la Universidad Politécnica de Madrid.

Gairín, J., Feixas, M., Guillamón, C., \& Quinquer, D. (2004). La tutoría académica en el escenario europeo de la Educación Superior. Revista interuniversitaria de formación del profesorado, 49, 61-78.

García Nieto, N., Asensio, I., Carballo, R., García García, M. y Guardia, S. (2005). La tutoría universitaria ante el proceso de armonización europea. Revista de educación, 337,189-210.

Nieto, N. G., Muñoz, I. I. A., Santaolalla, R. C., García, M. G., \& González, S. G. (2007). Guía para la labor tutorial en la Universidad en el Espacio Europeo de Educación Superior. http://www. mec. es/univ/proyectos2004/EA20040160. pdf em Dezembro de.

Rajadell, N. y Medina, A. (2009). Teorías de la enseñanza y del proceso formativo. En A. Medina y M.C. Domínguez (Eds.), Didáctica. Formación básica para profesionales de la educación (pp. 79-122). Madrid: Editorial Universitas, S.A.

Zabalza, M. (2003). Competencias docentes del profesorado universitario. Calidad y desarrollo profesional. Madrid: Narcea.

Zabalza, M. (2009). Ser profesor universitario hoy. La cuestión universitaria, 5, 69-81. 